\title{
ANÁLISE FÍSICA E AVALIAÇÃO DO EFEITO ANTIFÚNGICO DOS ÓLEOS DE ANDIROBA, COPAÍBA E PINHÃO-MANSO
}

\author{
Sara Freitas de Sousa ${ }^{1}$, Juarez Benigno Paes ${ }^{2 *}$, Marina Donária Chaves Arantes ${ }^{3}$, Yonny Martinez Lopez ${ }^{1}$, \\ Victor Fassina Brocco ${ }^{1}$ \\ ${ }^{1}$ Universidade Federal do Espírito Santo, Programa de Pós-Graduação em Ciências Florestais, Jerônimo Monteiro, Espírito Santo, Brasil. - \\ sara_freitas_stm@hotmail.com; jhonkar@gmail.com; vfbrocco@hotmail.com \\ 2*Universidade Federal do Espírito Santo, Departamento de Ciências Florestais e da Madeira, Jerônimo Monteiro, Espírito Santo, Brasil. - \\ Jbp2@uol.com.br \\ ${ }^{3}$ Universidade Federal de São João Del Rei, Departamento de Ciências Agrárias, Campus Sete Lagoas, Sete Lagoas, Minas Gerais, Brasil. - \\ mdonariac@hotmail.com
}

Recebido para publicação: 09/05/2017 - Aceito para publicação: 08/12/2017

\begin{abstract}
Resumo
Óleos de andiroba, copaíba e pinhão-manso são estudados por serem empregados em fins variados, como sabões, velas, medicamentos, biodiesel, tintas, fiação de lã e lustração de móveis. Também, são utilizados como inseticidas, fungicidas e moluscicidas. Objetivou-se neste trabalho caracterizar as propriedades físicas dos óleos de andiroba, copaíba e pinhão-manso, puros ou enriquecidos com iodo sublimado $\left(\mathrm{I}_{2}\right)$ e avaliar sua eficiência contra fungos de podridão parda e branca. Os óleos de andiroba e copaíba foram oriundos de comunidades do município de Santarém (PA) e o óleo de pinhão-manso, da Fazenda Tamanduá, município de Santa Terezinha (PB). Foram determinadas as características físicas ( $\mathrm{pH}$, índice de acidez, viscosidade e densidade) dos óleos e realizado o ensaio de toxidez em meio de cultura com fungos de podridão parda e branca. O enriquecimento dos óleos com iodo $\left(\mathrm{I}_{2}\right)$ proporcionou aumento da viscosidade. Foi constatada menor densidade para o óleo puro de andiroba e copaíba do que para o enriquecido com $5 \%$ de $\mathrm{I}_{2}$. O incremento de iodo nas soluções propiciou a diminuição de $\mathrm{pH}$ nos óleos de andiroba e copaíba, além do aumento do índice de acidez do óleo de andiroba. Para a avaliação da eficiência, os óleos foram empregados puros ou enriquecidos com iodo sublimado. No ensaio de toxidez em meio de cultura, para o fungo Trametes versicolor, a maior inibição em 20 dias foi para o tratamento com andiroba $1 \%$ de $\mathrm{I}_{2}$. Para o fungo Postia placenta, a maior inibição foi constatada para o tratamento com óleo de andiroba com $5 \%$ de $\mathrm{I}_{2}$.

Palavras-chave: Óleos vegetais, características físicas, toxidez, fungos xilófagos.
\end{abstract}

\begin{abstract}
Physical analysis and assessment of the antifungal effect of Carapa guianensis, Copaifera sp., and Jatropha curcas oils. Andiroba, or crabwood (Carapa guianensis), copaiba (Copaifera sp.) and jatropha (Jatropha curcas) oils are studied since they are used for a wide range of purposes, as soaps, candles, medicines, biodiesel, paints, wool spinning, and furniture lustration. Besides, they are also used as insecticides, fungicides, and molluscicides. The objective of this study was to characterize the physical properties of andiroba, copaíba and jatropha oils and to assess their efficiency against fungus of brown and white rot. The oils of andiroba and copaiba came from communities in the municipality of Santarém (state of Pará, Brazil), and the jatropha oil, from Fazenda Tamanduá, municipality of Santa Terezinha (state of Paraíba, Brazil). The physical characteristics ( $\mathrm{pH}$, acidity index, viscosity, and density) of the oils were determined, and the toxicity test was carried out in culture medium with brown rot fungi. The enrichment of the oils with iodine ( $\left.\mathrm{I}_{2}\right)$ provided higher viscosity. It was found lower density for pure andiroba and copaiba oil than for enriched oil with $5 \%$ I2. The addition of iodine in the solutions caused the decrease of $\mathrm{pH}$ value in the oils of andiroba and copaiba and the increase of acidity index on andiroba oil. For the assessment of efficiency, oils were tested either pure or enriched with sublimated iodine. For the toxicity test in culture medium, the greatest inhibition in 20 days occurred on the treatment of andiroba with $1 \%$ of $\mathrm{I}_{2}$ for Trametes versicolor fungus. For Postia placenta fungus, the highest inhibition was observed on the treatment of andiroba oil with $5 \%$ of $\mathrm{I}_{2}$.
\end{abstract}

Keywords: Vegetable oils, physical characteristics, toxicity, xylophagous fungi.

\section{INTRODUÇÃO}

Em função da quantidade escassa de madeiras que têm elevada durabilidade natural e da demanda do setor madeireiro, é de fundamental importância testar produtos que contribuam para a melhoria da resistência dessas madeiras a organismos xilófagos e que, também, sejam menos agressivos ao homem e ao ambiente,

FLORESTA, Curitiba, PR, v. 48, n. 2, p. 153-162, abr/jun 2018

Sousa. S. F. et.al.

ISSN eletrônico 1982-4688

DOI: $10.5380 /$ rf.v48 i2.52280 
quando comparados aos produtos de uso tradicional. Nesse sentido, pesquisas vêm sendo desenvolvidas para testar a eficiência de vários produtos com o intuito de proporcionar melhorias na resistência biológica da madeira a fungos e insetos (BRAND et al., 2006; BOSSARDI; BARREIROS, 2011; SOUZA; DEMENIGHI, 2017).

Os fungos apodrecedores são os principais organismos causadores de perdas em estruturas de madeira, como postes, dormentes e moirões. Para avaliar a resistência natural da madeira e a eficiência de substâncias químicas a organismos deterioradores, são necessários testes de laboratório e de campo. Esses ensaios consistem na submissão de amostras de madeira ao ataque de fungos causadores de podridão branca, parda ou mole, a insetos (térmitas e coleópteros) e a brocas marinhas.

Antes da realização dos ensaios com amostras de madeira, são empregados ensaios de triagem em meio de cultura a fim de se avaliar o potencial de toxidez dos produtos a serem testados contra os fungos xilófagos (MEDEIROS et al., 2016). Assim, vários produtos vêm sendo testados com esse intuito, com vista à obtenção de substâncias eficazes para impedir o desenvolvimento de fungos apodrecedores na madeira.

Com base nesses propósitos, os óleos vegetais, subprodutos de processos industriais e extratos naturais, estão sendo cada vez mais testados para o tratamento de madeira. Alguns exemplos são o óleo de candeia (Eremanthus erythropappus) (PAES et al., 2010) e seus subprodutos (TEIXEIRA et al., 2015), óleo de nim (Azadirachta indica) (PAES et al., 2012), Tall oil (BOSSARDI; BARREIROS, 2011) e extrativos do cerne de teca (BROCCO et al., 2017). Esses produtos naturais podem garantir a sustentabilidade do meio ambiente e a saúde humana, além de diminuir a utilização de produtos tradicionais, que possuem compostos tóxicos em sua formulação (TEIXEIRA et al., 2015).

Com base nas premissas elencadas e nas propriedades antifúngicas do iodo e sua baixa toxidez ao homem e ao ambiente (TAKAHASHI et al., 1985; IHSSEN et al., 2014), este trabalho teve como objetivos realizar a análise física dos óleos de andiroba, copaíba e pinhão-manso, puros ou enriquecidos com iodo sublimado $\left(\mathrm{I}_{2}\right)$ e avaliar sua eficiência contra o crescimento e desenvolvimento de fungos causadores de podridão parda e branca.

\section{MATERIAL E MÉTODOS}

\section{Procedência, enriquecimento e análise física dos óleos}

Os óleos utilizados foram provenientes de regiões distintas. Os óleos de andiroba (Carapa guianensis Aubl.) e copaíba (Copaifera sp.) foram oriundos de comunidades do município de Santarém (PR) e o óleo de pinhão-manso (Jatropha curcas L.) foi cedido pela Fazenda Tamanduá, no município de Santa Terezinha (PB). Tais produtos foram utilizados logo que recebidos no Laboratório de Biodeterioração e Proteção da Madeira (LBPM) - Departamento de Ciências Florestais e da Madeira (DCFM) da Universidade Federal do Espírito Santo (UFES), em Jerônimo Monteiro (ES). Para atender aos objetivos da pesquisa, os óleos foram usados puros ou enriquecidos com iodo sublimado $\left(\mathrm{I}_{2}\right)$ de acordo com a Tabela 1. Todas as análises foram realizadas em março de 2016.

Tabela 1. Óleos puros ou enriquecidos com iodo utilizados em cada tratamento.

Table 1. Pure or enriched with iodine oils used in each treatment.

\begin{tabular}{cc}
\hline Tratamento & Óleo Puro ou Enriquecido \\
\hline 1 & Andiroba Puro \\
2 & Andiroba enriquecido com $1 \%$ de Iodo \\
3 & Andiroba enriquecido com 3\% de Iodo \\
4 & Andiroba enriquecido com 5\% de Iodo \\
5 & Copaíba Puro \\
6 & Copaíba enriquecido com 1\% de Iodo \\
7 & Copaíba enriquecido com 3\% de Iodo \\
8 & Copaíba enriquecido com 5\% de Iodo \\
9 & Pinhão-manso Puro \\
10 & Pinhão-manso enriquecido com 1\% de Iodo \\
11 & Pinhão-manso enriquecido com 3\% de Iodo \\
12 & Pinhão-manso enriquecido com 5\% de Iodo \\
\hline
\end{tabular}

Para verificar as propriedades dos óleos e soluções utilizadas (enriquecimento com $\mathrm{I}_{2}$ ), foram determinados o índice de acidez (IA), pH, densidade e viscosidade. Para a determinação do IA, conforme proposto por Oliveira et al. (2012) e Adorno et al. (2013), $5 \mathrm{~g}$ de óleo foram dissolvidos em $50 \mathrm{~mL}$ de etanol 100\%, sendo adicionado o indicador fenolftaleína. Sob agitação constante, realizou-se a titulação em $\mathrm{KOH}$ a $0,5 \mathrm{~mol} \mathrm{~L}^{-1}$. 
Os resultados do índice de acidez foram obtidos pela Equação 1, citada por Oliveira et al. (2012) e Sampaio et al. (2012).

$$
\mathrm{A}=\frac{\mathrm{V} \times \mathrm{f} \times \mathrm{N} \times 56,1}{\mathrm{~m}}
$$

Em que: $\mathrm{A}=$ Índice de acidez $\left(\mathrm{mg} \mathrm{KOH} \mathrm{g}^{-1}\right) ; \mathrm{N}=$ Normalidade do titulante ( $\left.\mathrm{mol} \mathrm{l}^{-1}\right)$; $\mathrm{V}=$ Volume utilizado $(\mathrm{mL})$; $\mathrm{F}=$ Fator de correção da solução de $\mathrm{KOH}(1,1606)$; e $\mathrm{m}=$ Massa da amostra $(\mathrm{g})$.

$\mathrm{O}$ pH das soluções foi determinado por meio de pHmetro digital. A viscosidade dos óleos puros foi determinada em condições de laboratório. Os óleos de copaíba e andiroba enriquecidos com iodo sublimado $\left(\mathrm{I}_{2}\right)$ foram aquecidos a $40{ }^{\circ} \mathrm{C}$ por 10 minutos, a fim de reduzir a viscosidade e permitir leituras. Foi utilizado um viscosímetro rotacional digital para leitura direta da análise. Para a determinação da densidade das soluções, foram utilizados picnômetros de $25 \mathrm{~mL}$, conforme metodologia citada por Paes et al. (2015) ao dividir a massa de óleo pelo seu respectivo volume. Os valores de índice de acidez, $\mathrm{pH}$, densidade e viscosidade dos óleos foram verificados em cinco replicas (alíquotas) e avaliados por estatística descritiva, em função da média aritmética e coeficiente de variação, uma vez que as tomadas das amostras foram do mesmo recipiente e não consideradas com repetições, o que impossibilitou a análise de variância.

\section{Ensaio de toxidez em meio de cultura}

Para este ensaio, foi utilizado o método descrito por Medeiros et al. (2016). Esse método consiste em colocar o óleo em poço de $0,6 \mathrm{~cm}$ de diâmetro, perfurado no centro de placa de Petri, contendo meio de cultura, para o desenvolvimento do fungo. Para tanto, foram adicionados $20 \mathrm{~mL}$ de meio em cada placa, preparado pela dissolução de $15 \mathrm{~g}$ de ágar e $20 \mathrm{~g}$ de maltose em $1 \mathrm{~L}$ de água destilada, conforme metodologia descrita na American Society for Testing and Materials - ASTM D - 1413 (2018). Foram adicionados 0,5\% do óleo em cada placa.

Os óleos foram empregados puros ou enriquecidos com $\mathrm{I}_{2}$ nas proporções de 1, 3 e $5 \%$ em relação ao volume de óleo (Tabela 1). Foram dispostos em placas a fim de se atingir uma retenção de 50 a $70 \mathrm{~kg} \cdot \mathrm{m}^{-3}$, definida com base em Paes et al. (2010) para a proteção da madeira de sumaúma (Ceiba pentandra) contra cupins subterrâneos. Os fungos (inóculo de 1 x $1 \mathrm{~cm}$ ) foram dispostos em duas extremidades opostas da placa de Petri. Foram utilizadas culturas puras dos fungos Postia placenta [(Fr.) M.J. Larsen \& Lombard)], (Madison 698), podridão parda e Trametes versicolor (L.: Fr.) Pilát, (Madison 697), podridão branca, pertencentes ao LBPM/DECF/UFES. O ensaio foi mantido em sala climatizada $\left(28 \pm 2{ }^{\circ} \mathrm{C}\right.$ e $\left.75 \pm 5 \%\right)$ até o completo desenvolvimento dos fungos nas placas controle, contendo apenas o meio de cultura.

Foram avaliadas cinco repetições para cada fungo, óleo e enriquecimento testados. Para avaliar o crescimento dos fungos, foram obtidas fotografias, analisadas no software ImagePro® Plus, version 4.5.0.29 para Windows 98/NT/2000, com a finalidade de definir a área colonizada pelo fungo e a ação do produto testado. Os dados foram avaliados por estatística descritiva em função da média aritmética e coeficiente de variação, uma vez que os mesmos não tiveram distribuição normal (teste de Lilliefors) e as variâncias não foram homogêneas (teste de Cochran). Sendo assim, não foram permitidos a análise de variâncias e o teste de comparação entre médias.

\section{RESULTADOS}

\section{Avaliação física dos óleos}

Foram verificadas menores densidades nos óleos puros de andiroba e copaíba do que nos enriquecidos com $5 \%$ de iodo $\left(\mathrm{I}_{2}\right)$ (Tabela 2). O oposto ocorreu para o óleo de pinhão-manso, que teve diminuição da densidade com o aumento da concentração de $\mathrm{I}_{2}$. O menor valor encontrado foi para o enriquecido com $3 \%$. Nota-se que houve aumento da viscosidade dos óleos com a adição do iodo, sendo constatado valor de até 385,30 MPa.s ${ }^{-1}$ para o óleo de copaíba enriquecido com $5 \%$ de $\mathrm{I}_{2}$. 
Tabela 2. Densidade, viscosidade, índice de acidez e pH dos óleos puros ou enriquecidos com iodo.

Table 2. Density, viscosity, acidity, and $\mathrm{pH}$ values for pure or enriched with iodine oils.

\begin{tabular}{|c|c|c|c|c|}
\hline \multirow{3}{*}{ Óleo } & \multicolumn{4}{|c|}{ Densidade $\left({\left.\mathrm{g} . \mathrm{cm}^{-3}\right)}^{-3}\right.$} \\
\hline & \multirow{2}{*}{ Puro } & \multicolumn{3}{|c|}{ Proporções de Iodo (\%) } \\
\hline & & 1 & 3 & 5 \\
\hline \multirow[t]{2}{*}{ Andiroba } & 1,022 & 0,971 & 0,973 & 1,075 \\
\hline & $(2,85)$ & $(0,13)$ & $(0,27)$ & $(0,49)$ \\
\hline \multirow[t]{2}{*}{ Copaíba } & 1,044 & 1,110 & 1,025 & 1,062 \\
\hline & $(4,85)$ & $(0,15)$ & $(0,10)$ & $(5,27)$ \\
\hline \multirow[t]{2}{*}{ Pinhão-manso } & 1,021 & 1,055 & 0,957 & 1,002 \\
\hline & $(3,56)$ & $(0,29)$ & $(0,29)$ & $(0,27)$ \\
\hline \multirow{3}{*}{ Óleo } & \multicolumn{4}{|c|}{ Viscosidade $\left(\right.$ MPa.s $^{-1}$ ou cP) } \\
\hline & \multirow{2}{*}{ Puro } & \multicolumn{3}{|c|}{ Proporções de Iodo (\%) } \\
\hline & & 1 & 3 & 5 \\
\hline \multirow[t]{2}{*}{ Andiroba } & 65,54 & 69,38 & 104,12 & 108,52 \\
\hline & $(5,65)$ & $(1,83)$ & $(4,63)$ & $(7,15)$ \\
\hline \multirow[t]{2}{*}{ Copaíba } & 67,46 & 123,84 & 203,06 & 385,30 \\
\hline & $(9,54)$ & $(1,06)$ & $(2,73)$ & $(3,61)$ \\
\hline \multirow[t]{2}{*}{ Pinhão-manso } & 67,60 & 79,02 & 94,53 & 97,26 \\
\hline & $(2,11)$ & $(4,78)$ & $(0,26)$ & $(2,14)$ \\
\hline \multirow{3}{*}{ Óleo } & \multicolumn{4}{|c|}{ Índice de Acidez (mg KOH.g ${ }^{-1}$ ) } \\
\hline & \multirow{2}{*}{ Puro } & \multicolumn{3}{|c|}{ Proporções de Iodo (\%) } \\
\hline & & 1 & 3 & 5 \\
\hline \multirow[t]{2}{*}{ Andiroba } & 68,36 & 76,50 & 86,27 & 120,45 \\
\hline & $(3,61)$ & $(4,09)$ & $(4,92)$ & $(20,53)$ \\
\hline \multirow[t]{2}{*}{ Copaíba } & 111,66 & 117,19 & 115,57 & 111,01 \\
\hline & $(3,05)$ & $(27,70)$ & $(3,59)$ & $(1,92)$ \\
\hline \multirow[t]{2}{*}{ Pinhão-manso } & 87,57 & 39,06 & 58,92 & 76,17 \\
\hline & $(1,55)$ & $(16,49)$ & $(6,19)$ & $(6,74)$ \\
\hline \multirow{3}{*}{ Óleo } & \multicolumn{4}{|c|}{ pH } \\
\hline & \multirow{2}{*}{ Puro } & \multicolumn{3}{|c|}{ Proporções de Iodo (\%) } \\
\hline & & 1 & 3 & 5 \\
\hline \multirow[t]{2}{*}{ Andiroba } & 3,3 & 2,2 & 2,1 & 1,6 \\
\hline & $(0,00)$ & $(0,18)$ & $(0,19)$ & $(0,21)$ \\
\hline \multirow[t]{2}{*}{ Copaíba } & 4,8 & 2,8 & 3,1 & 3,1 \\
\hline & $(8,84)$ & $(0,02)$ & $(0,03)$ & $(0,03)$ \\
\hline \multirow[t]{2}{*}{ Pinhão-manso } & 3,9 & 4,0 & 4,1 & 4,1 \\
\hline & $(7,25)$ & $(0,00)$ & $(0,01)$ & $(0,01)$ \\
\hline
\end{tabular}

Valores entre parênteses são os coeficientes de variação (\%).

Observa-se que quanto maior a concentração de iodo, mais ácida a solução se tornou para o óleo de andiroba. A diminuição do $\mathrm{pH}$ das soluções enriquecidas pode ser explicada pelo valor do $\mathrm{pH}$ do óleo puro (ácido), assim como pela adição do $\mathrm{I}_{2}$, utilizado para o enriquecimento e que também possui caráter ácido. $\mathrm{O}$ óleo de pinhão-manso teve comportamento diferenciado, tendo aumento de $\mathrm{pH}$ conforme o incremento das concentrações de iodo. Isso pode ter ocorrido, possivelmente, devido a alguma característica tampão do produto.

Os valores obtidos para as medidas de $\mathrm{pH}$ indicam que todos os óleos utilizados são ácidos ( $\mathrm{pH}<7)$, com variação de 3,3 a 4,8 para os óleos puros, sendo o óleo de andiroba o mais ácido. Com relação ao índice de acidez

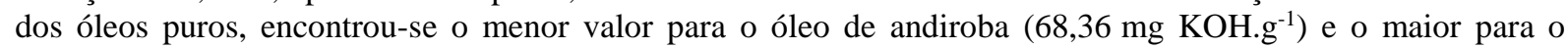
de opaíba $\left(111,66 \mathrm{mg} \mathrm{KOH} . \mathrm{g}^{-1}\right)$.

\section{Toxidez em meio de cultura}

Para o fungo Trametes versicolor, submetidom ao tratamento com óleo de andiroba, houve diminuição do crescimento com a adição do iodo (10 dias) (Tabela 3). Após 20 dias, observou-se valor de $14,52 \mathrm{~cm}^{2}$ para esse óleo puro e $9,73 \mathrm{~cm}^{2}$ para o óleo enriquecido com $5 \%$ de $\mathrm{I}_{2}$ (Tratamentos 1 e 4). 
Tabela 3. Área de crescimento e inibição dos fungos Trametes versicolor e Postia placenta para os tratamentos testados.

Table 3. Area of growth and inhibition of Trametes versicolor and Postia placenta fungi for the treatments tested.

\begin{tabular}{|c|c|c|c|c|}
\hline \multirow{3}{*}{ Tratamento } & \multicolumn{4}{|c|}{ Fungo Trametes versicolor } \\
\hline & \multicolumn{2}{|c|}{ Área de Crescimento $\left(\mathrm{cm}^{2}\right)$} & \multicolumn{2}{|c|}{ Inibição do Crescimento Fúngico (\%) } \\
\hline & 10 dias & 20 dias & 10 dias & 20 dias \\
\hline 1 & $* *$ & 14,52 & $* *$ & 53,41 \\
\hline 2 & 4,26 & 5,11 & 74,23 & 83,62 \\
\hline 3 & 3,30 & 7,19 & 80,05 & 76,94 \\
\hline 4 & 2,69 & 9,73 & 83,76 & 68,78 \\
\hline 5 & 2,84 & 17,28 & 82,87 & 44,58 \\
\hline 6 & 9,08 & 15,07 & 45,13 & 51,66 \\
\hline 7 & $* *$ & 12,13 & $* *$ & 61,09 \\
\hline 8 & 8,14 & 11,52 & 50,79 & 63,04 \\
\hline 9 & 6,61 & 63,58 & 60,08 & - \\
\hline 10 & 12,50 & 63,58 & 24,44 & - \\
\hline 11 & 9,51 & 11,40 & 42,52 & 63,43 \\
\hline 12 & 4,49 & 9,68 & 72,89 & 68,96 \\
\hline Controle & 16,54 & 63,58 & - & - \\
\hline
\end{tabular}

\begin{tabular}{|c|c|c|c|c|}
\hline \multirow{3}{*}{ Tratamento } & \multicolumn{4}{|c|}{ Fungo Postia placenta } \\
\hline & \multicolumn{2}{|c|}{ Área de crescimento $\left(\mathrm{cm}^{2}\right)$} & \multicolumn{2}{|c|}{ Inibição do crescimento fúngico (\%) } \\
\hline & 10 dias & 20 dias & 10 dias & 20 dias \\
\hline 1 & 10,75 & 18,93 & 21,55 & 39,26 \\
\hline 2 & 9,57 & 17,28 & 30,21 & 44,58 \\
\hline 3 & 7,69 & 11,76 & 43,86 & 62,27 \\
\hline 4 & 3,98 & 6,51 & 70,99 & 79,10 \\
\hline 5 & 13,15 & 13,72 & 4,07 & 55,98 \\
\hline 6 & 12,92 & 14,39 & 5,77 & 53,85 \\
\hline 7 & 12,25 & 17,50 & 10,60 & 43,87 \\
\hline 8 & 11,04 & 15,69 & 19,42 & 49,66 \\
\hline 9 & 10,46 & 14,66 & 23,65 & 52,98 \\
\hline 10 & 9,24 & 14,39 & 32,60 & 53,85 \\
\hline 11 & 6,97 & 12,44 & 49,11 & 60,09 \\
\hline 12 & 5,11 & 11,52 & 62,74 & 63,04 \\
\hline Controle & 13,70 & 63,58 & - & - \\
\hline
\end{tabular}

Controle, placa contendo apenas o meio de cultura. Em que: Tratamento 1: óleo de andiroba puro; Tratamento 2: óleo de andiroba com 1\% de $\mathrm{I}_{2}$; Tratamento 3: óleo de andiroba com 3\% de $\mathrm{I}_{2}$; Tratamento 4: óleo de andiroba com 5\% de $\mathrm{I}_{2}$; Tratamento 5: óleo de copaíba puro; Tratamento 6: óleo de copaíba com $1 \%$ de $\mathrm{I}_{2}$; Tratamento 7: óleo de copaíba com 3\% de $\mathrm{I}_{2}$; Tratamento 8: óleo de copaíba com 5\% de $\mathrm{I}_{2}$; Tratamento 9: óleo de pinhão-manso puro; Tratamento 10: óleo de pinhão-manso com $1 \%$ de $\mathrm{I}_{2}$; Tratamento 11: óleo de pinhão-manso com 3\% de $\mathrm{I}_{2}$; Tratamento 12: óleo de pinhão-manso com $5 \%$ de $\mathrm{I}_{2}$. **: Sem possibilidade de observação; -: Ausência de inibição.

Para Postia placenta, os valores registrados para área de crescimento do fungo submetido a soluções (óleo puro e enriquecidos com 1,3 e $5 \%$ de $\mathrm{I}_{2}$ ) foram inferiores aos da placa controle (Tabela 3 ). A placa controle estava completamente colonizada na última observação (20 dias, com área de $63,58 \mathrm{~cm}^{2}$ ), sendo utilizada para avaliar a potencialidade de desenvolvimento dos fungos.

\section{DISCUSSÃO}

\section{Análises físicas dos óleos}

Os valores de densidade dos óleos puros de andiroba e pinhão-manso encontrados nesta pesquisa foram semelhantes (Tabela 2). Segundo Ribeiro e Seravalli (2004), a densidade de um óleo está associada ao grau de ligações duplas ou triplas existentes em suas moléculas, o que possibilita relação inversamente proporcional. Sendo assim, quanto menor o peso molecular do óleo, maior é o grau de instauração. Com base nesse argumento, os óleos testados possuem graus de instauração similares.

Para as propriedades físicas dos óleos de pinhão-manso, Beltrão e Oliveira (2008) citam densidade de $0,88 \mathrm{~g} . \mathrm{cm}^{-3}$ a $15^{\circ} \mathrm{C}$ e viscosidade cinemática de 4,016 centistokes (cSt) determinada a $40{ }^{\circ} \mathrm{C}$. A maior densidade

FLORESTA, Curitiba, PR, v. 48, n. 2, p. 153-162, abr/jun 2018

Sousa. S. F et al.

ISSN eletrônico 1982-4688

DOI: $10.5380 /$ rf.v48 i2.52280 
encontrada para o óleo utilizado nesta pesquisa pode estar associada à temperatura $\left(25^{\circ} \mathrm{C}\right)$ e técnica utilizada para sua obtenção, que, neste caso, foi o uso de picnómetros.

Para as propriedades físico-químicas do óleo-resina de copaíba de árvore de duas áreas distintas da Floresta Nacional do Tapajós - FLONA, foram constatadas densidade média de 0,96 g.cm-3 e viscosidade de 82,27 MPa.s (SILVA et al., 2012). O valor de densidade obtido na presente pesquisa foi superior ao obtido pelos autores citados e, o de viscosidade, inferior. As características físico-químicas dos óleos podem variar entre e dentre indivíduos, região e período de frutificação das árvores. Segundo Melo et al. (2014), a viscosidade é variável nos óleos vegetais, sendo dependente do grau de insaturação e tamanho das cadeias graxas da sua composição; além de também ser influenciado pela presença de ramificações e posicionamento dessas insaturações.

Em estudo realizado por Alves et al. (2015), no qual foram analisadas as características físico-químicas

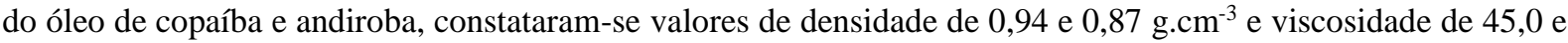
18,3 $\mathrm{MPa} . \mathrm{s}^{-1}$, respectivamente. A viscosidade do óleo de andiroba (18,3 $\left.\mathrm{MPa}^{-1}{ }^{-1}\right)$ relatada foi, aproximadamente, três vezes menor do que a obtida pela presente pesquisa $\left(65,54 \mathrm{MPa}^{-1}{ }^{-1}\right)$. Tais diferenças podem estar associadas a fatores já citados anteriormente por Melo et al. (2014).

A viscosidade está relacionada à penetração do líquido na madeira, pois quanto maior for seu valor, menor será a penetração, estando relacionada com sua facilidade de impregnar a parede celular. Assim, soluções menos viscosas não necessitariam ser diluídas ou aquecidas para penetrarem e se difundirem no lenho (PAES et al., 2015).

Em pesquisa realizada por Vasconcelos et al. (2008), foi encontrado $\mathrm{pH}$ de 4,5 para o óleo de Copaifera multijuga, valor próximo ao obtido na presente pesquisa (Tabela 2). Tal valor indica que o óleo tem caráter ácido.

Para o índice de acidez (massa de hidróxido de potássio necessária na neutralização dos ácidos livres presentes em um grama de amostra de óleo), foi verificado que os maiores índices encontrados foram os do óleo

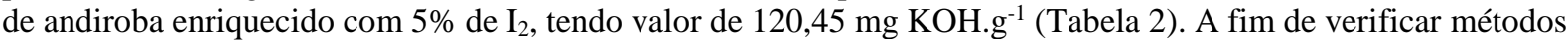

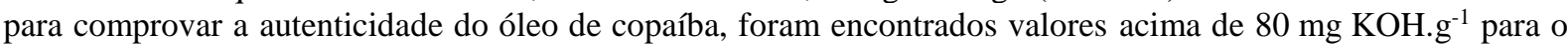
índice de acidez deste óleo e de misturas com álcool etílico. Produtos que possuem valores abaixo do citado podem ser considerados adulterados (VASCONCELOS; GODINHO, 2002). Sendo assim, o óleo utilizado nesta pesquisa tinha boa procedência.

Para o IA do óleo de andiroba, Silva et al. (2012) constaram valores de 9,62 a 10,17 mg KOH.g ${ }^{-1}$,

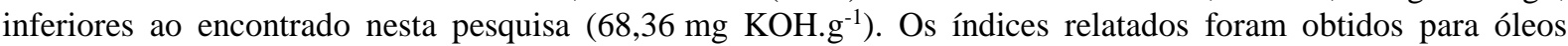
extraídos de plantas da Floresta Nacional do Tapajós, Pará. Tal diferença pode estar associada a fatores já citados anteriormente, com base em Melo et al. (2014).

O óleo de pinhão-manso possuiu tendência diferente para o índice de acidez quando comparado aos demais. Inicialmente, o produto puro teve IA de $87,57 \mathrm{mg} \mathrm{KOH.g}{ }^{-1}$ e, quando enriquecido com iodo a $5 \%$, diminuiu para $76,17 \mathrm{mg} \mathrm{KOH} \cdot \mathrm{g}^{-1}$ (Tabela 2). Não foi obtida, na literatura, nenhuma informação sobre o comportamento dos óleos utilizados em relação à adição de iodo, bem como informações sobre as características avaliadas para o óleo de pinhão-manso.

\section{Ensaio de toxidez em meio de cultura}

Houve dificuldade na observação do micélio de Trametes versicolor nos dez primeiros dias de colonização no meio de cultura para os tratamentos 1 e 7 (Tabela 3), pois suas hifas eram hialinas. Mesmo assim, na primeira observação do experimento (após 10 dias), o menor valor de área de crescimento foi encontrado para o óleo de andiroba enriquecido com $5 \%$ de iodo, sendo correspondente a 2,69 $\mathrm{cm}^{2}$. Logo, a inibição do crescimento fúngico foi maior $(83,76 \%)$ para esse tratamento.

Constatou-se valor alto de inibição de crescimento (82,87\%) para o óleo de copaíba puro (Tratamento 5) aos 10 dias de observação, demonstrando, também, sua eficiência. Porém, na segunda verificação (20 dias), essa inibição diminuiu para 44,58\%, pois o crescimento do fungo foi maior, o que normalmente ocorre quando não há inibição total do crescimento.

Os tratamentos 9 e 10, que correspondem ao óleo de pinhão-manso puro e enriquecido com $1 \%$ de $\mathrm{I}_{2}$, respectivamente (Tabela 3), demonstraram pouca eficiência, pois a placa já estava completamente colonizada na segunda observação (20 dias). Porém, em concentrações maiores de iodo nesse óleo (3 e 5\%), notou-se eficiência, uma vez que houve inibição do crescimento fúngico (Tratamentos 11 e 12). A área de crescimento das placas controles (testemunha) foi superior à de todos os valores encontrados nos tratamentos. As testemunhas encontravam-se completamente colonizadas no vigésimo dia.

De modo geral, observou-se que os valores das médias de crescimento para Postia placenta foram diminuindo conforme o enriquecimento dos óleos com iodo. Logo, foi aumentada a inibição desse fungo, demonstrando, assim, a eficácia e propriedades fungicidas deste elemento $\left(\mathrm{I}_{2}\right)$, exceto para o óleo de copaíba aos 20 dias do ensaio (Tratamentos 5; 6; 7 e 8) (Tabela 3). A maior área de crescimento no décimo dia foi a da colônia submetida ao óleo de copaíba puro. Logo, foi o menos eficiente por ter a menor inibição fúngica $(4,07 \%)$. 
Porém, essa tendência não persistiu aos 20 dias do ensaio, sendo a maior colonização a do óleo de andiroba puro $\left(18,93 \mathrm{~cm}^{2}\right)$, o que representou 39,26\% de inibição do crescimento fúngico.

$\mathrm{O}$ tratamento 4 (óleo de andiroba $+5 \%$ de $\mathrm{I}_{2}$ ) proporcionou a menor área de crescimento nos dois períodos de leitura. Na visualização das placas em 20 dias, a média de crescimento foi, aproximadamente, 10 vezes menor que o controle $\left(63,58 \mathrm{~cm}^{2}\right)$. Notou-se que, com o passar dos dias, as colônias aumentaram o diâmetro ou persistiram com a mesma área colonizada. Mesmo assim, não foram superiores quando comparadas ao controle.

Os valores encontrados para inibição do crescimento foram crescentes de 10 a 20 dias de leitura, uma vez que, para gerar estes valores, levou-se em consideração a área colonizada da placa controle, que teve valor de $13,70 \mathrm{~cm}^{2}$ aos 10 dias e, na última leitura, obteve valor maior, com a placa completamente colonizada $\left(63,58 \mathrm{~cm}^{2}\right)$. $\mathrm{O}$ óleo de pinhão-manso enriquecido com 5\% de iodo (Tratamento 13) também foi um bom inibidor do crescimento desse fungo, com valores de inibição de 62,74 e 63,04\% em 10 e 20 dias, respectivamente.

Os fungos Trametes versicolor (podridão branca) e Postia placenta (podridão parda) possuem sistemas enzimáticos diferentes. Dessa forma, atacaram os constituintes dos óleos empregados na pesquisa de modo diferenciado. Na madeira, os fungos de podridão branca têm a capacidade de degradarem a lignina, enquanto os de podridão parda degradam apenas os polissacarídeos (LEPAGE et al., 2017).

Não constam informações sobre a inibição de fungos dos óleos testados nesta pesquisa na literatura. Porém, Medeiros et al. (2016), ao testarem o efeito inibitório dos óleos essenciais de três plantas do Cerrado (Lippia origanoides, Lippia lacunosa e Syzygium aromaticum) aos fungos Gloeophyllum trabeum (podridão parda) e Trametes versicolor, observaram que o óleo de Lippia origanoides teve a maior atividade fungicida para os dois fungos dentre os óleos testados e que o de Lippia lacunosa teve eficiência apenas contra o Trametes versicolor.

Em trabalho realizado por Brocco et al. (2017), foi testada a eficiência do extrato do cerne da madeira de teca com 20 anos de idade, obtido em água quente nas concentrações de 0,125, 0,25, 050, 1, 2, 4 e 8\% em relação ao meio de cultura. Foi observado aumento na inibição do crescimento do fungo Postia placenta na medida em que se incrementou a concentração do extrato no meio. A concentração de $4 \%$ inibiu seu crescimento completamente.

Alguns trabalhos citam o potencial do iodo no controle de fungos xilófagos, obtendo resultados promissores. Também, enfatizam que esse produto é recomendado por possuir baixa toxidez ao homem e a animais domésticos e alto efeito inibitório (TAKAHASHI et al., 1985). Produtos com iodo, utilizados no tratamento de madeira, além de controlar, devem também inibir o desenvolvimento de organismos xilófagos. No entanto, como enfatizam Medeiros et al. (2016), testes em nível de campo são necessários para avaliar a real potencialidade dos óleos naturais a organismos xilófagos.

\section{CONCLUSÕES}

As análises realizadas permitem concluir que:

- O enriquecimento dos óleos com iodo $\left(\mathrm{I}_{2}\right)$ proporcionou aumento da viscosidade. Os óleos de andiroba puro e de copaíba enriquecido com $5 \%$ de $\mathrm{I}_{2}$ tiveram menor densidade.

- $\quad$ incremento de iodo nas soluções propiciou a diminuição do pH nos óleos de andiroba e copaíba e aumento do índice de acidez no óleo de andiroba.

- No ensaio de toxidez com Trametes versicolor, a maior inibição, aos 20 dias, foi a do óleo de andiroba com $1 \%$ de $\mathrm{I}_{2}$. Para Postia placenta, a maior inibição foi a do óleo de andiroba com $5 \%$ de $\mathrm{I}_{2}$.

- O ensaio de laboratório indicou a eficiência dos produtos. Porém, para comprovar sua verdadeira eficácia, são necessários ensaios de campo.

\section{REFERÊNCIAS}

AMERICAN SOCIETY FOR TESTING AND MATERIALS. ASTM D-1413: Standard test method for wood preservatives by laboratory soil-block cultures. Annual Book of ASTM Standard, Philadelphia, 2008, 7p.

ADORNO, W. T.; ALMEIDA, L. J.; MARTINS, G. A. S.; ALVES, D. G.; SOUSA, J. P. Avaliação do índice de acidez do óleo bruto de soja adicionado de LCC natural, visando produção de biodiesel. UNOPAR Científica. Ciências Biológicas e da Saúde, Londrina, v. 15, n. 4, p. 283-286, 2013.

ALVES, W. F.; SOUZA, M. C.; ALMEIDA, A. N. S.; OLIVEIRA, S. S.; RIBEIRO I. L. R. Características físicoquímicas de óleos essenciais de plantas da região do vale do Juruá. Enciclopédia Biosfera, Goiânia, v.11, n.22, p. 1-13, 2015.

FLORESTA, Curitiba, PR, v. 48, n. 2, p. 153-162, abr/jun 2018

Sousa. S. F. et.al.

ISSN eletrônico 1982-4688

DOI: $10.5380 /$ rf.v48 i 2.52280 
BELTRÃO, N. E. M.; OLIVEIRA M. I. P. Oleaginosas e seus óleos: vantagens e desvantagens para produção de biodiesel. Campina Grande: Embrapa Algodão, 2008, 28p. (Documentos, 201).

BOSSARDI, K.; BARREIROS, R. M. Produtos naturais como preservantes para madeiras de rápido crescimento - uma revisão. Ciência da Madeira, Pelotas, v. 2, n. 2, p. 109-118, 2011.

BROCCO, V. F.; PAES, J. B.; COSTA, L. G.; BRAZOLIN, S.; ARANTES, M. D. C. Potential of teak heartwood extracts as a natural wood preservative. Journal of Cleaner Production, Knoxville, v. 142, n. 4, p. 2093-2099, 2017.

IHSSEN, J.; SCHUBERT, M.; THÖNY-MEYER, L.; RICHTER, M. Laccase catalyzed synthesis of iodinated phenolic compounds with antifungal activity. Plos One, San Francisco, v. 9, n. 3, p. 1-12, 2014

LEPAGE, E.; SALIS, A. G.; GUEDES, E. C. R. Tecnologia de proteção da madeira. São Paulo: Montana Química, 2017.225p.

MEDEIROS, F. C. M.; GOUVEIA, F. N.; BIZZO, H. R.; VIEIRA, R. F.; DEL MENEZZI, C. H. S. Fungicidal activity of essential oils from Brazilian Cerrado species against wood decay fungi. International Biodeterioration \& Biodegradation, Berlin, v. 114, p. 87-93, 2016.

MELO, M. A. M. F.; MELO, M. A. R.; PONTES, A. S, G. C. FARIAS, A. F. F.; DANTAS, M. B.; CALIXTO, C. D.; SOUZA, A. G.; CARVALHO FILHO, J.R. Non-conventional oils for biodiesel production: a study of thermal and oxidative stability. Journal of Thermal Analysis and Calorimetry, New York, 117, n, 2, p. 845849, 2014.

OLIVEIRA, D. S.; FONSECA, X. D. S.; FARIAS, P. N.; BEZERRA, V. S.; PINTO, C. H. C.; SOUZA, L. D.; SANTOS, A. G. D.; MATIAS, L. G. O. Obtenção do biodiesel através da transesterificação do óleo de Moringa oleifera Lam. Holos, Natal, v. 1, n. 28, p. 49-61, 2012.

PAES, J. B.; SOUZA, A. D.; LIMA, C. R.; SANTANA, G. M. Rendimento e características físicas dos óleos de nim (Azadirachta indica) e mamona (Ricinus communis). Floresta e Ambiente, Seropédica, v. 22, n. 1, p.134139, 2015.

PAES, J. B.; SOUSA, A. D.; LIMA, C. R.; SOUZA, P. F. Eficiência dos óleos de nim (Azadirachta indica) e mamona (Ricinus communis) na resistência da madeira de sumaúma (Ceiba pentandra) a fungos xilófagos em simuladores de campo. Ciência Florestal, Santa Maria, v. 22, p. 617-624, 2012.

PAES, J. B.; FONSÊCA, C. M. B.; LIMA, C. R.; SOUZA, A. D. Eficiência do óleo de candeia na melhoria da resistência da madeira de sumaúma a cupins. Cerne, Lavras, v. 16, n. 2, p. 217-225, 2010.

RIBEIRO, E. P., SERAVALLI, E. A. G. Química de alimentos. São Paulo: Edgard Blücler, Instituto Mauá de Tecnologia, 2004. 184 p.

SAMPAIO, C. P.; ARAÚJO R. F.; SANTOS, S. B.; DONZELES; S. M. L. Avaliação das propriedades físicas e da qualidade do óleo de sementes de pinhão-manso (Jatropha curcas L.) armazenadas. Engenharia na Agricultura, Viçosa, v. 20, n.1, p. 12-18, 2012.

SILVA, E. S.; MATHIAS, C. S.; LIMA, M. C. F.; VEIGA JUNIOR, V. F.; RODRIGUES, D. P.; CLEMENT, C. R. Análise físico-química do óleo-resina e variabilidade genética de copaíba na Floresta Nacional do Tapajós. Pesquisa Agropecuária Brasileira, Brasília, v. 47, n. 11, p. 1621-1628, 2012.

SOUZA, R. V.; DEMENIGHI, A. L. Tratamentos preservantes naturais de madeiras de floresta plantada para a construção civil. Mix Sustentável, Florianópolis, v.3, n.1, p. 84-92, 2017.

TAKAHASHI, M.; TSUNODA, K.; IMAMURA, Y.; ADACHI, A.; NISHIMOTO, K. Studies on the production of preservative-treated plywood. (1) Preservative effectiveness of glue-line treated plywood with low toxicity chemicals. Mokuzai Hozon (Wood Preservation), Mokuzai, v. 11, n. 2, p. 11-24. 1985.

TEIXEIRA, J. G.; LATORRACA, J. V. F.; TREVISAN, H.; PAES, J. B. Eficiência do óleo de neem e dos resíduos de candeia sobre a inibição do desenvolvimento de fungos xilófagos. Scientia Forestalis, Piracicaba, v. 43, n. 106, p. 417-426, 2015.

VASCONCELOS, A. F. F.; GODINHO, O. E S. Uso de métodos analíticos convencionados no estudo da autenticidade do óleo de copaíba. Química Nova, São Paulo, v. 25, n. 6, p. 1057-1060, 2002. 
VASCONCELOS, K. R. F.; VEIGA JUNIOR, V. F. ROCHA, W. C.; BANDEIRA, M. F. C. L. Avaliação in vitro da atividade antibacteriana de um cimento odontológico à base de óleo-resina de Copaifera multijuga Hayne. Revista Brasileira de Farmacognosia, Curitiba, v. 18, Suplemento, p. 733-738, 2008.

FLORESTA, Curitiba, PR, v. 48, n. 2, p. 153-162, abr/jun 2018

Sousa. S. F. et.al.

ISSN eletrônico 1982-4688

DOI: $10.5380 /$ rf.v48 i2.52280 
FLORESTA, Curitiba, PR, v. 48, n. 2, p. 153-162, abr/jun 2018 Sousa. S. F. et.al. 